\title{
Statistical Analysis for Generalized Progressive Hybrid Censored Data from Lindley Distribution under Step-Stress Partially Accelerated Life Test Model
}

\author{
Aakriti Pandey \\ Arun Kaushik \\ Sanjay K. Singh \\ Department of Statistics, Institute of Science, \\ Banaras Hindu University, Varanasi, India-221005.
}

Umesh Singh

\begin{abstract}
The aim of this paper is to present the estimation procedure for the step-stress partially accelerated life test model under the generalized progressive hybrid censoring scheme. The uncertainties are assumed to be governed by Lindley distribution. The problem with point and interval estimation of the parameters as well as the acceleration factor using maximum likelihood approach for the step-stress partially accelerated life test model has been considered. A simulation study is conducted to monitor the performance of the estimators on the basis of the mean squared error under the considered censoring scheme. The expected total time of the test under an accelerated condition is computed to examine the effects of the parameters on the duration of the test. In addition, a graph of the expected total time of the test under accelerated and un-accelerated conditions is provided to highlight the effect due to acceleration. One real data set has been analyzed for illustrative purposes.
\end{abstract}

Keywords: accelerated life test, generalized progressive hybrid censoring, maximum likelihood estimates, total expected number of failures, total time of the test.

\section{Introduction}

In recent years, the rapid increase in the popularity of the accelerated life test (ALT) is due to its wide range of applicability. To sustain the pressure of the global competitive market, significant efforts have been made by manufacturers to develop products to comply with industry standards, satisfying high reliabilities as per customers' need. Today, the challenge is to evaluate reliabilities of said products under time and cost constraints. The ALT is a turning point in tackling such problems by applying additional load (stress) to the products to shorten the testing period and provide information about lifetime distribution more quickly. In accelerated testing conditions, the product is subjected to more a severe environment compared to the typical operating environment, which triggers the failures sooner, with the purpose of quantifying the life characteristics of the products.

In ALTs, all units are subjected to higher than usual stress levels. Conversely, in a modified 
ALT, which is referred to as the partial accelerated life test (PALT), units are tested at both accelerated and normal conditions. The goal of PALTs is to gather more failure data within a limited time without applying high stress to all test units. The PALTs are extensively used in areas with time deficits and those that are of an economical nature. The information obtained by such tests can be utilized to estimate the failure behavior of the units under normal conditions. A proper statistical modelling of PALTs was conducted by DeGroot and Goel 1979, where the authors considered the tempered random variable model for PALTs. Nelson 1990 suggested a multitude of ways in which stress under accelerated conditions can be applied. The most popular among these are constant stress and step stress. The choice of stress loading depends on the nature of the unit utilized in the service, as well as other limitations. In constant stress accelerated tests, each unit is exposed to fixed stress until a pre-specified time or upto the occurrence of the fixed number of failures. In step stress accelerated tests, the units are subjected to successive high levels of stresses at prefixed times or upto the occurrence of the fixed number of failures. A test consisting of two stress levels, one that starts with a normal stress level at a fixed point of time and changes to other stress level, is referred to as a step stress partially accelerated life test(SSPALT).

In life testing experiments, censoring is a common feature that may occur naturally or is the result of a constraint. Among the conventional Type-I, Type-II, and hybrid censoring schemes, the latter, which was introduced by Epstein (1954), is quite popular in life testing experiments. The details of statistical inferences and applications for the exponential distribution under hybrid censoring is presented in Balakrishnan (1996) and Childs et al. (2003). In conventional censoring schemes, the surviving units can be removed only at the terminal point of the experiment. However, certain situations exists wherein the removal of live units are not localized, except for the terminal points of the experiment, but the allowance of intermediate removals are equally important. Kundu and Joarder (2006) and Childs et al. (2008) have considered the Type-I progressive hybrid censoring scheme(Type-I PHCS) in the context of life testing experiments in which $n$ identical units are tested with progressive censoring schemes $\left(R_{1}, R_{2}, \cdots, R_{m}\right)$ and the experiment is terminated at time $T^{*}=\min \left\{X_{m: m: n}, T\right\}$, where $T \in$ $(0, \infty)$ and $1 \leq m \leq n$ are fixed in advance and $X_{1: m: n} \leq X_{2: m: n} \leq \cdots$ are the observed ordered failure times from the experiment. One can navigate through Balakrishnan and Aggarwala (2000) and Balakrishnan (2007) for the observations regarding the detailed description of the censoring scheme. The advantage of this censoring scheme is that the termination time is fixed, i.e., the test can never go beyond $T$. In this way, this scheme controls both the time and cost of the experiment. However, if the unknown average lifetime is relatively high compared to the stopping time, then there is the possibility of fewer than $m$ failures to be observed, eventually reducing the efficiency of the inferences based on the censored data. Keeping this point in mind, Childs et al. (2008) proposed the Type-II progressive hybrid censoring scheme that terminates the experiment at time $T^{*}=\max \left\{X_{m: m: n}, T\right\}$. Hence, it ensures a minimum of $m$ number of failures in the data set and therefore, ensures the efficiency to be at a more desired level. When $X_{m: m: n}>T$, the experiment is terminated at $m$ th failure with withdrawals occurring at each failure as per the pre-specified progressive scheme $\left(R_{1}, R_{2}, \cdots, R_{m}\right)$. A consequence of this would be that it may lead to significant increase in the time of termination. On the other hand, when $X_{m: m: n}<T$, we observe failure upto time $T$. If $D$ is the number of failures that occur before time $T$, then the progressive censoring scheme, in this case, is $\left(R_{1}, R_{2}, \cdots, R_{m}, R_{m+1}, \cdots, R_{D}\right)$, where $R_{m}=R_{m+1}=\cdots=R_{D}=0$. Thus, we see that the termination time in this censoring scheme is random and unknown to the experimenter. From the above discussion, we may note that the Type-I hybrid censoring scheme keeps the termination time of the experiment below the prefixed value by forgoing the efficiency where as Type-II hybrid censoring ensures efficiency more than the prefixed level but foregoes the termination time. Therefore, the need for a censoring scheme controlling both the termination time and efficiency was felt which should ensure the bare minimum number of failures and place restrictions on time. Cho et al. (2015) introduced the generalized progressive hybrid (GPH) censoring scheme, which assimilates the features of both (Type-I 
PHC and Type-II PHC), as well as irons-out their lacunas.

Bai and Chung 1992 estimated the scale parameter and the acceleration factor for exponential distribution based on Type-I censored sample using the method of maximum likelihood. AbdElfattah et al. 2008 used the maximum likelihood approach to estimate the parameters of the Burr type-XII distribution and the acceleration factor based on Type-I censored sample under SSPALTs. Ismail (2012) obtained the maximum likelihood estimates of the parameters of Weibull distribution and the acceleration factor from hybrid partially accelerated life test. A continuum to the above, we have used the method of maximum likelihood to estimate the parameter of Lindley distribution and the acceleration factor with generalized progressive hybrid(GPH) censoring scheme under SSPALTs.

This present paper is comprised of eight sections. Section 2 describes the considered censoring scheme. In section 3, the considered model is discussed. Section 4 provides point and interval estimates based on maximum likelihood. In section 5, a simulation study is conducted to study the performance of the estimators in terms of mean squared error(MSE). In section 6 , the expected total time of the test is evaluated. Section 7 presents the analysis of one real data set and discussions. Finally, a conclusion has been provided in section 8 .

\section{The censoring scheme}

Suppose $n$ units are put on test. Keeping in mind the desired minimum efficiency and cost of the items, we prefix integers $k, m \in\{1,2, \cdots, n\}$ such that $k<m$. The lifetime of the sample units $X_{1}, X_{2}, \cdots, X_{n}$ are independent and identically distributed random variables from a distribution with cumulative density function (cdf) $F(\cdot)$ and probability density function (pdf) $f(\cdot) . R_{i}$ units are randomly removed at $i^{t h}$ failure, satisfying the condition $\sum_{i=1}^{m} R_{i}+m=n$. The test continues until, the stopping time $T^{*}=\max \left\{X_{k: m: n}, \min \left\{X_{m: m: n}, T\right\}\right\}$ is reached, and, remaining units are removed from the experiment. It may be noted that instead of observing $\mathrm{m}$ number of failures, this scheme guarantees a minimum number of $k$ failures. Let, $D$ denote the number of observed failures up to time $T$. Thus, three cases arise in this scheme and we have set of observations as given below:

$$
\begin{array}{rll}
\text { Case-I: } & X_{1: m: n}, \cdots, X_{2: m: n}, \cdots, X_{k: m: n}, \quad \text { if } T<X_{k: m: n}, \\
\text { Case-II: } & X_{1: m: n}, \cdots, X_{k: m: n}, \cdots, X_{D: m: n}, \quad \text { if } X_{k: m: n}<T<X_{m: m: n}, \\
\text { Case-III: } & X_{1: m: n}, \cdots, X_{k: m: n}, \cdots, X_{m: m: n}, \quad \text { if } X_{k: m: n}<X_{m: m: n}<T .
\end{array}
$$

A pictorial representation of this censoring scheme is given in Figure 1. Note that for CaseI, $X_{k+1: m: n}, \cdots, X_{m: m: n}$ are not observed; likewise, for Case-II, $X_{D+1: m: n}, \cdots, X_{m: n: n}$ are not observed. Given a generalized progressive censored sample, the likelihood functions for Case-I, Case-II and Case-III denoted by $L_{I}, L_{I I}, \& L_{I I I}$ respectively are given below:

$$
\begin{aligned}
& \text { Case-I: } \quad L_{I}(\epsilon, \zeta)=K_{1} \prod_{j=1}^{k-1} f\left(x_{j: m: n}\right)\left[1-F\left(x_{j: m: n}\right)\right]^{R_{j}} f\left(x_{k: m: n}\right)\left[1-F\left(x_{k: m: n}\right)\right]^{R_{k}^{*}} \\
& \text { Case-II: } L_{I I}(\epsilon, \zeta)=K_{2} \prod_{j=1}^{D} f\left(x_{j: m: n}\right)\left[1-F\left(x_{j: m: n}\right)\right]^{R_{j}}[1-F(T)]^{R_{D+1}^{*}}, \\
& \text { Case-III: } L_{I I I}(\epsilon, \zeta)=K_{3} \prod_{j=1}^{m} f\left(x_{j: m: n}\right)\left[1-F\left(x_{j: m: n}\right)\right]^{R_{j}},
\end{aligned}
$$

where $K_{1}=\prod_{j=1}^{k} \sum_{k=j}^{m}\left(R_{k}+1\right), K_{2}=\prod_{j=1}^{D} \sum_{k=j}^{m}\left(R_{k}+1\right), K_{3}=\prod_{j=1}^{m} \sum_{k=j}^{m}\left(R_{k}+1\right), R_{k}^{*}=$ $n-k-\sum_{i=1}^{k-1} R_{i}$ and $R_{D+1}^{*}=n-D-\sum_{i=1}^{D} R_{i}$. 
Number of removals

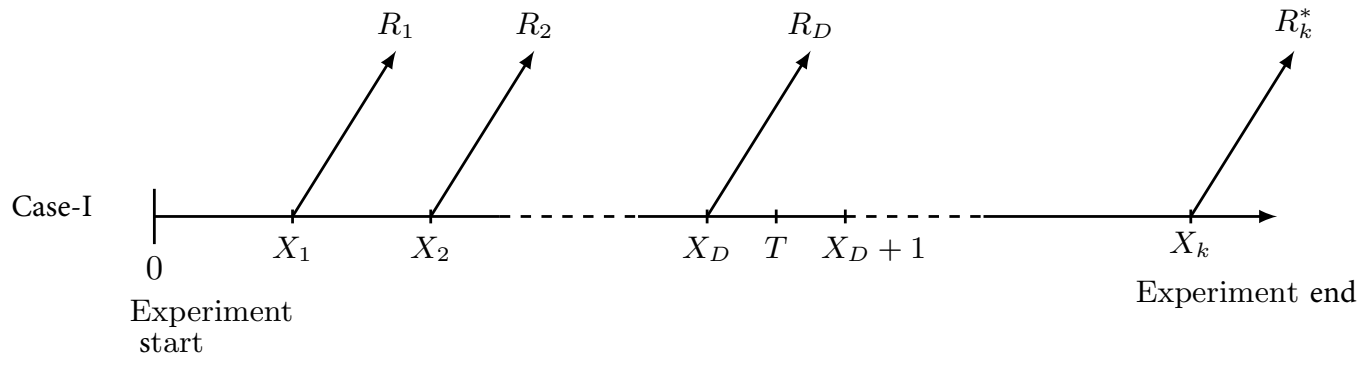

Number of removals

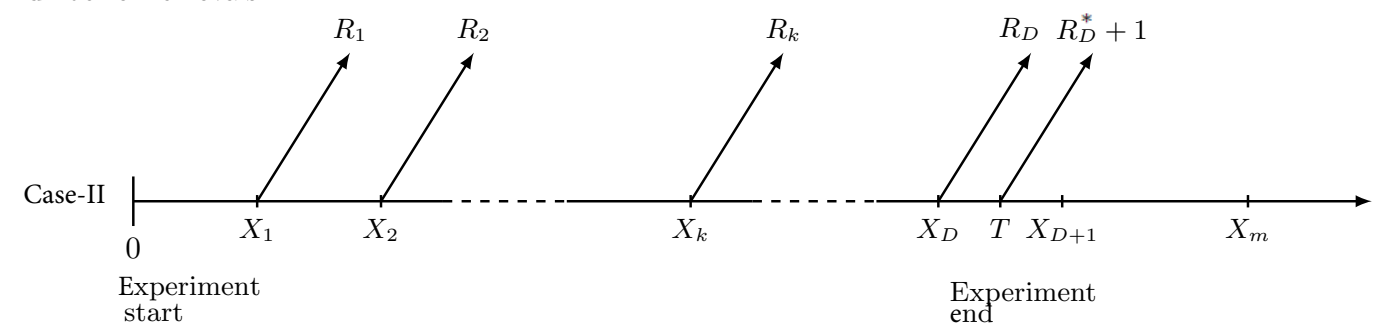

Number of removals

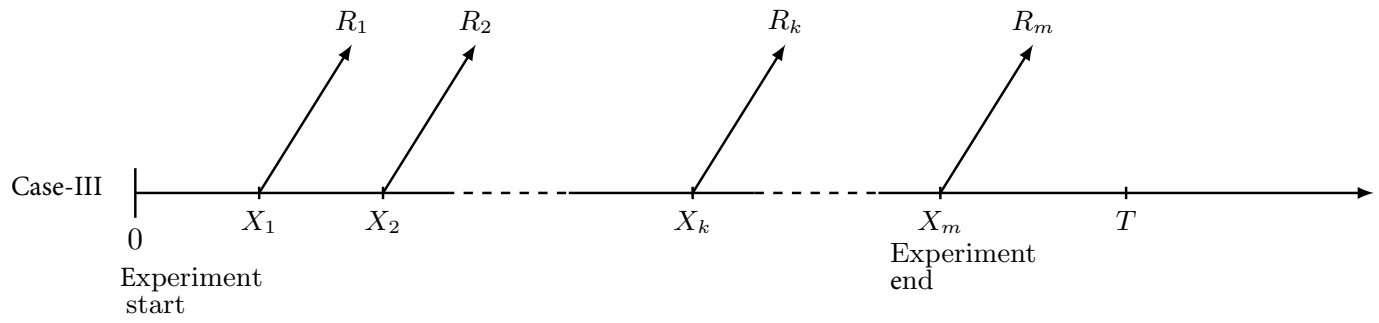

Figure 1: Schematic representation of the generalized progressive hybrid censoring scheme

\section{The model description}

The article considers point and interval estimation of Lindley distribution parameter under SSPALTs with the GPH censoring scheme. Lindley distribution was first introduced by Lindley (1958) to analyze lifetime data. Lindley distribution can be considered as mixture of exponential and gamma distribution. It has the ability to model lifetime data with an increasing, decreasing, unimodal, and bath tub shaped hazard rate. One may navigate through Cakmakyapan and Ozel (2016) for detailed overview of the properties of Lindley distribution. He has suggested that it often performs better than the traditional exponential distribution. Taking cognisance of the facts discussed above, we are motivated to consider this model in the present article.

Let the lifetime of each experimental item, i.e., $X$ follows Lindley distribution with parameter $\epsilon$. The pdf of one parameter Lindley distribution is given by

$$
f(x)=\frac{\epsilon^{2}}{(1+\epsilon)}(1+x) e^{-\epsilon x} ; x>0, \epsilon>0
$$

and the corresponding distribution function is given by

$$
F(x)=\left[1-\left(1+\frac{\epsilon x}{1+\epsilon}\right) e^{-\epsilon x}\right] ; x>0, \epsilon>0
$$

\subsection{Assumptions}

1. The lifetimes of the items follow Lindley distribution with parameter $\epsilon$. 
2. The total lifetime $X$ of an item is defined as

$$
X=\left\{\begin{array}{ll}
T, & 0<T \leq \tau \\
\tau+\zeta^{-1}(T-\tau), & t>\tau
\end{array},\right.
$$

where $T$ is the lifetime of the items at normal stress, $\tau$ is the time at which the stress is to be increased and $\zeta$ is the acceleration factor.

3. The lifetimes of the test items are independent and identically distributed random variable.

4. Under the GPH censoring scheme, the test terminates at $\max \left\{X_{k: m: n}, \min \left\{X_{m: m: n}, T\right\}\right\}$.

\subsection{Experimental procedure}

1. Initially $n$ identical and independent items are put on test and subjected to normal stress conditions.

2. The stress level is changed at prefixed time $\tau$ and observe the failure time of the items until the termination time $\max \left\{X_{k: m: n}, \min \left\{X_{m: m: n}, T\right\}\right\}$ is reached.

3. As soon as the experiment starts, the items start experiencing the risk of failing. At the time of ith failure, $R_{i}$ units are randomly removed, satisfying the condition $\sum_{i=1}^{m} R_{i}+m=n$. At the time of termination the remaining items are removed from the experiment. Under the above mentioned GPH censoring scheme one of the following samples will be observed:

(a) $x_{1: m: n}<x_{2: m: n}<\cdots<x_{n_{u}: m: n} \leq \tau<x_{n_{u+1}: m: n}<\cdots<x_{1: m: n}$, if $T<X_{k: m: n}$.

(b) $x_{1: m: n}<x_{2: m: n}<\cdots<x_{n_{u}: m: n} \leq \tau<x_{n_{u+1}: m: n}<\cdots<x_{D: m: n}$, if $X_{k: m: n}<T<$ $X_{m: m: n}$.

(c) $x_{1: m: n}<x_{2: m: n}<\cdots<x_{n_{u}: m: n} \leq \tau<x_{n_{u+1}: m: n}<\cdots<x_{m: m: n}$, if $X_{k: m: n}<$ $X_{m: m: n}<T$.

The pdf of random variable $X$ under step stress partially accelerated life test is given by

$$
f(x)= \begin{cases}0, & x \leq 0 \\ f^{1}(x), & 0<x \leq \tau, \\ f^{2}(x), & x>\tau\end{cases}
$$

where $f^{1}(x)$, the PDF of $X$ before acceleration time $\tau$ will be equivalent to $f(x)$ given in 2 and $f^{2}(x)$, the PDF of $x$ after acceleration time $\tau$ will be

$$
f^{2}(x)=\zeta \frac{\epsilon^{2}}{(1+\epsilon)}[1+(\tau+\zeta(x-\tau))] e^{-\epsilon(\tau+\zeta(x-\tau))} ; x>\tau, \epsilon>0 .
$$

Similarly, $F^{1}(x)$, the CDF of $X$ before acceleration time $\tau$ will be equivalent to $F(x)$ given in 3 and $F^{2}(x)$, the CDF of $X$ after acceleration time $\tau$ given in the following equation:

$$
F^{2}(x)=\left[1-\left(1+\frac{\epsilon(\tau+\zeta(x-\tau))}{1+\epsilon}\right) e^{-\epsilon(\tau+\zeta(x-\tau))}\right] ; x>0, \epsilon>0 .
$$




\section{Estimation process}

\subsection{Point and interval estimation}

The present section deals with point and interval estimation of the parameter of the considered model based on the data obtained from the experiment explained above i.e. GPH censored data under SSPALT. The likelihood function is given by

$$
L(\underset{\sim}{x}, \epsilon, \zeta) \propto \prod_{i=1}^{n_{u}} f^{1}\left(x_{i}\right)\left[1-F^{1}\left(x_{i}\right)\right]^{R_{i}} \prod_{i=n_{u}+1}^{J} f^{2}\left(x_{i}\right)\left[1-F^{2}\left(x_{i}\right)\right]^{R_{i}} W(\epsilon, \zeta)
$$

Where $F^{1}(\cdot)$ and $F^{2}(\cdot)$ are the CDFs before and after acceleration time $\tau$ and $W(\epsilon, \zeta)$ is defined as

$$
W(\epsilon, \zeta)= \begin{cases}1, & \text { if } J=k, m \\ {\left[\left\{1+\frac{\epsilon(\tau+\zeta(T-\tau))}{1+\epsilon}\right\} e^{-\epsilon(\tau+\zeta(T-\tau))}\right]^{R_{D+1}^{*}},} & \text { if } J=D .\end{cases}
$$

Substituting $f^{1}\left(x_{i}\right), f^{2}\left(x_{i}\right)$ and corresponding CDFs from equation(2), equation(3), equation(6) and equation(7) in equation(8), we have

$$
\begin{aligned}
&L \underset{\sim}{x}, \epsilon, \zeta) \propto(\zeta)^{J-n_{u}}\left[\frac{\epsilon^{2}}{1+\epsilon}\right]^{J} e^{-\epsilon \sum_{i=1}^{n_{u}} x_{i}} \prod_{i=1}^{n_{u}}\left(1+x_{i}\right)\left[\left(1+\frac{\epsilon x_{i}}{1+\epsilon}\right) e^{-\epsilon x_{i}}\right]^{R_{i}} \times \\
& \prod_{i=n_{u}+1}^{J}\left[1+\left(\tau+\zeta\left(x_{i}-\tau\right)\right)\right] e^{-\epsilon\left(\tau+\zeta\left(x_{i}-\tau\right)\right)\left(1+R_{i}\right)} \\
& {\left[1+\frac{\epsilon\left(\tau+\zeta\left(x_{i}-\tau\right)\right)}{1+\epsilon}\right]^{R_{i}} W(\epsilon, \zeta) . }
\end{aligned}
$$

Thus, the corresponding log likelihood function is

$$
\begin{aligned}
\ln L= & \left(J-n_{u}\right) \ln (\zeta)+J \ln \left(\frac{\epsilon^{2}}{1+\epsilon}\right)-\epsilon \sum_{i=1}^{n_{u}} x_{i}+\sum_{i=1}^{n_{u}} \ln \left(1+x_{i}\right) \\
& +\sum_{i=1}^{n_{u}} R_{i} \ln \left[\left(1+\frac{\epsilon x_{i}}{1+\epsilon}\right) e^{-\epsilon x_{i}}\right]+\sum_{i=n_{u}+1}^{J} \ln \left[1+\left(\tau+\zeta\left(x_{i}-\tau\right)\right)\right] \\
& -\epsilon \sum_{i=n_{u}+1}^{J}\left(\tau+\zeta\left(x_{i}-\tau\right)\right)\left(1+R_{i}\right)+\sum_{i=n_{u}+1}^{J} R_{i} \ln \left[1+\frac{\epsilon\left(\tau+\zeta\left(x_{i}-\tau\right)\right)}{1+\epsilon}\right] \\
& +\ln W(\epsilon, \zeta) .
\end{aligned}
$$

The first order partial derivatives of the above equation with respect to $\epsilon$ and $\zeta$ are obtained and equated to zero to get the likelihood equations which are given below:

$$
\begin{gathered}
\frac{\partial \ln L}{\partial \zeta}=\frac{\left(J-n_{u}\right)}{\zeta}+\sum_{i=n_{u}+1}^{J} \frac{\left(x_{i}-\tau\right)}{\left[1+\left(\tau+\zeta\left(x_{i}-\tau\right)\right)\right]}-\epsilon \sum_{i=n_{u}+1}^{J}\left(x_{i}-\tau\right)\left(1+R_{i}\right) \\
+\sum_{i=n_{u}+1}^{J} R_{i} \frac{\left(x_{i}-\tau\right) \frac{\epsilon}{1+\epsilon}}{\left[1+\frac{\epsilon\left(\tau+\zeta\left(x_{i}-\tau\right)\right)}{1+\epsilon}\right]}+\frac{\partial \ln W(\epsilon, \zeta)}{\partial \zeta}=0 \\
\frac{\partial \ln L}{\partial \epsilon}=J \frac{\epsilon+2}{\epsilon(\epsilon+1)}-\sum_{i=1}^{n_{u}} x_{i}+\sum_{i=1}^{n_{u}} R_{i}\left[-\epsilon+\frac{x_{i}}{(1+\epsilon)\left(1+\epsilon\left(1+x_{i}\right)\right)}\right] \\
\sum_{i=1}^{n_{u}} R_{i}\left[\frac{\left(\tau+\zeta\left(x_{i}-\tau\right)\right)}{(1+\epsilon)^{2}}\right]+\frac{\partial \ln W(\epsilon, \zeta)}{\partial \epsilon}=0 .
\end{gathered}
$$


Since the analytical solution of these equations is not possible, it is suggested to find a numerical solution. The exact expression for MLEs is not obtainable, the exact distribution of MLEs is also not obtainable. In such a case the large sample theory is proved to be the strategic imperative to obtain the confidence interval. Let $I(\zeta, \epsilon)$ be the Fisher's information matrix denoted as

$$
I(\zeta, \epsilon)=\left[\begin{array}{cc}
-E\left(\frac{\partial^{2} \ln L}{\partial \zeta^{2}}\right) & -E\left(\frac{\partial^{2} \ln L}{\partial \zeta \partial \epsilon}\right) \\
-E\left(\frac{\partial^{2} \ln L}{\partial \zeta \partial \epsilon}\right) & -E\left(\frac{\partial^{2} \ln L}{\partial \epsilon^{2}}\right)
\end{array}\right] .
$$

Then, $I^{-1}(\hat{\zeta}, \hat{\epsilon})$ will be the estimated variance-covariance matrix and diagonal elements of this matrix provide the estimated asymptotic variances for the acceleration factor $\zeta$ and the parameter $\epsilon$. The two sided $100(1-\kappa) \%$ asymptotic confidence intervals of $\zeta$ and $\epsilon$ can, therefore, be defined as $\hat{\zeta} \pm z_{\kappa / 2} \sqrt{\operatorname{var}(\hat{\zeta})}$ and $\hat{\epsilon} \pm z_{\kappa / 2} \sqrt{\operatorname{var}(\hat{\epsilon})}$. Here, $\operatorname{var}($.$) denotes the$ asymptotic variance. We have used the $\operatorname{optim}(\cdot)$ function available in R-software to find the ML estimates.

\section{Simulation studies}

The present section elaborates the simulation study with the motto of checking the performance of estimators in terms of their mean square errors(MSEs). Here, it may be noted that the MSEs of the estimators will depend on the values of $n, k, m$ and $T$, and hence, various choices have been made to study the effect thereof. In addition to the above, the estimated $95 \%$ asymptotic confidence intervals of the model parameter and the accelerating factor are also computed. We have considered the following four removal patterns in order to study the effect of pattern of removal on the estimation of the parameter and the acceleration factor:

$S_{m: n}{ }^{(1)}:$ All the removals are at the last observation, i.e., $R_{m}=\mathrm{n}-\mathrm{m}$.

$S_{m: n}{ }^{(2)}$ :All the removals are done at the first failure, i.e., $R_{1}=\mathrm{n}-\mathrm{m}$.

$S_{m: n}{ }^{(3)}$ :The removals are at first and the last observation, i.e., $R_{1}=R_{m}=(\mathrm{n}-\mathrm{m}) / 2$.

$S_{m: n}{ }^{(4)}$ :The removals are at middle observations, i.e., $R_{m / 2}=R_{m / 2+1}=(\mathrm{n}-\mathrm{m}) / 2$.

\section{Algorithm: Simulation of GPH censored data}

1. Generate $k$ independent standard uniform random variables $U_{1}, U_{2}, \cdots, U_{k}$.

2. Compute $B_{i}=U^{1 / \gamma_{i}} ; i=1, \cdots, k$, where $\gamma_{i}=n-i+1+\sum_{j=i}^{m} R_{j}$.

3. Start with $V_{0}=1$ and compute $V_{i}=B_{i} V_{i-1}$ and $U_{r: m: n}=1-V_{r} \forall r=1, \cdots, k$.

4. If $U_{k: m: n}>F(T)$, then Goto Step-6, Else $l=k+1$ and Goto Step-5.

5. While $V_{l-1}>1-F(T)$ and $l \leq m$ Do:

(a) Generate a uniform random variable $\mathrm{U}$.

(b) Define $B_{l}=U^{\frac{1}{\sum_{i=l}^{m} R_{i}+1}}$ and $V_{l}=V_{l-1} * B_{l}$.

(c) $\mathrm{l}=\mathrm{l}+1$.

6. $X_{j}^{(I I)}=F^{\leftarrow}\left(1-V_{j}\right) ; j=1, \cdots, l$, and Stop.

Here $F^{\leftarrow}(\cdot)$ is the inverse cdf function defined as $F^{\leftarrow}(p)=\inf \{x \in R: F(x) \geq p\}$. 


\section{Expected total time of the test}

The expected total time of a test (ETTT) is defined as the expected time required to complete the experiment. An experimenter may wish to explore the experimental time duration for selecting an appropriate sampling plan. The cost and time duration to complete a test are interrelated. A significant high ETTT ultimately results into a high cost of conducting an experiment. So, it is quite economical and practical to have a glimpse of the time required to complete the experiment for a particular life test plan. The ETTT for the considered scheme is defined as

$$
\begin{aligned}
E(T T T)= & T \times \operatorname{Pr}\left[X_{k: m: n}<T<X_{m: m: n}\right]+E\left(X_{m: m: n} \mid X_{m: m: n}<T\right) \\
& \times \operatorname{Pr}\left[X_{m: m: n}<T\right]+E\left[X_{k: m: n} \mid T<X_{k: m: n}\right] \operatorname{Pr}\left[T<X_{k: m: n}\right],
\end{aligned}
$$

where,

$$
\operatorname{Pr}\left[X_{k: m: n}<T<X_{m: m: n}\right]=\sum_{j=k}^{m-1} \operatorname{Pr}\left[X_{j}<T<X_{j+1}\right] .
$$

Following Eissa et al. (2014), the expression for $P\left(X_{j}<T<X_{j+1}\right)$ can be obtained as:

$$
\begin{aligned}
\operatorname{Pr}\left[X_{j}<T<X_{j+1}\right] & =\int_{-\infty}^{\tau}\left(1-F_{x_{j+1}}^{1}\left(T_{L} \mid x_{j}=x\right)\right) f_{x_{j}}^{1}(x) d x \\
& +\int_{\tau}^{T}\left(1-F_{x_{j+1}}^{2}\left(T_{L} \mid x_{j}=x\right)\right) f_{x_{j}}^{2}(x) d x \\
& =\text { (A) }+ \text { (B) } \quad \text { (say) },
\end{aligned}
$$

where $F^{1}(),. f^{1}($.$) and F^{2}(.) f^{2}($.$) are the \mathrm{CDF}$ and PDF before and after acceleration time $\tau$. By putting $F^{1}(),. f^{1}($.$) and F^{2}(.) f^{2}($.$) from equation(2), equation(3), equation(6) and$ equation(7) in the equation(14), (A) can be easily expressed as

$$
\begin{aligned}
\text { (A) }= & c_{j-1} \sum_{i=1}^{j} a_{i, j} \frac{\epsilon^{2}}{1+\epsilon}\left(\left(1+\frac{\epsilon \tau}{1+\epsilon}\right) e^{-\epsilon \tau}\right)^{\gamma_{j}+1} \sum_{i^{\prime}=0}^{\gamma_{i}-\gamma_{j}-2}\left(\frac{\epsilon}{1+\epsilon}\right)^{i^{\prime}}\left(\begin{array}{c}
\gamma_{i}-\gamma_{j}-2 \\
i^{\prime}
\end{array}\right) \\
& {\left[\left(\frac{1}{\epsilon\left(\gamma_{i}-\gamma_{j}-1\right)}\right)^{i^{\prime}+1} \gamma\left(i^{\prime}+1,\left(\epsilon \tau\left(\gamma_{i}-\gamma_{j}-1\right)\right)\right)+\left(\frac{1}{\epsilon\left(\gamma_{i}-\gamma_{j}-1\right)}\right)^{i^{\prime}+2} \gamma\left(i^{\prime}+2,\left(\epsilon \tau\left(\gamma_{i}-\gamma_{j}-1\right)\right)\right)\right] }
\end{aligned}
$$

where $\gamma(\cdot)$ is lower incomplete gamma integral defined as $\gamma(s, x)=\int_{0}^{x} t^{s-1} e^{-t} d t, \gamma_{j}=n-j+$ $1+\sum_{i=j}^{m} R_{i} ; 1 \leq j \leq m, c_{j-1}=\prod_{i=1}^{j} \gamma_{i} ; 1 \leq j \leq m$ and $a_{i, j}=\prod_{k \neq i=1}^{j} \frac{1}{\gamma_{k}-\gamma_{i}} ; 1 \leq i \leq j \leq m$. Similarly, integral (B) can be solved as follow:

$$
\begin{aligned}
\text { (B) }= & c_{j-1} \sum_{i=1}^{j} a_{i, j} \frac{\epsilon^{2}}{1+\epsilon}\left(\left(1+\frac{\epsilon \tau^{\prime}}{1+\epsilon}\right) e^{-\epsilon \tau^{\prime}}\right)^{\gamma_{j}+1} \sum_{j^{\prime}=0}^{\gamma_{i}-\gamma_{j}-2}\left(\begin{array}{c}
\gamma_{i}-\gamma_{j}-2 \\
j^{\prime}
\end{array}\right) \\
& \left(\frac{\epsilon}{1+\epsilon}\right)^{j^{\prime}}\left[\left(\frac{1}{\epsilon\left(\gamma_{i}-\gamma_{j}-1\right)}\right)^{j^{\prime}+1}\left[\gamma\left(j^{\prime}+1, b^{\prime}\right)-\gamma\left(j^{\prime}+1, a^{\prime}\right)\right]\right. \\
+ & \left.\left(\frac{1}{\epsilon\left(\gamma_{i}-\gamma_{j}-1\right)}\right)^{j^{\prime}+2}\left[\gamma\left(j^{\prime}+2, b^{\prime}\right)-\gamma\left(j^{\prime}+2, a^{\prime}\right)\right]\right],
\end{aligned}
$$

where $\tau^{\prime}=\tau+\zeta(T-\tau), b^{\prime}=\epsilon \tau^{\prime}\left(\gamma_{i}-\gamma_{j}-1\right)$ and $a^{\prime}=\tau \epsilon\left(\gamma_{i}-\gamma_{j}-1\right)$. It is well known that

$$
f_{x_{k}}\left(x \mid X_{k: m: n}>T\right)=\frac{f_{X_{k}}(x)}{\operatorname{Pr}\left[X_{k: m: n}>T\right]} \quad \& \quad f_{x_{m}}\left(x \mid X_{m: m: n}<T\right)=\frac{f_{X_{m}}(x)}{\operatorname{Pr}\left[X_{m: m: n}<T\right]} .
$$


Hence, conditional expectation of $X_{m}$ will be

$$
\begin{aligned}
E\left(X_{m} \mid X_{m: m: n}<T\right) & =\int_{0}^{T} x \frac{f_{X_{m}}(x)}{\operatorname{Pr}\left[X_{m: m: n}<T\right]} d x \\
& =\frac{\int_{0}^{\tau} x f_{X_{m}}^{1}(x) d x+\int_{\tau}^{T} x f_{X_{m}}^{2}(x) d x}{\operatorname{Pr}\left[X_{m: m: n}<T\right]} \\
& =\frac{\Phi+\varnothing)}{\operatorname{Pr}\left[X_{m: m: n}<T\right]} \quad \text { (say). }
\end{aligned}
$$

Now, after solving the respective integrals, expressions for $(\otimes)$ and $(\bar{Y}$ are given below:

$$
\begin{aligned}
\Phi & =\epsilon c_{m-1} \sum_{i=1}^{m} a_{i, m} \sum_{k^{\prime}=0}^{\gamma_{i}-1}\left(\frac{\epsilon}{1+\epsilon}\right)^{k^{\prime}+1}\left(\begin{array}{c}
\gamma_{i}-1 \\
k^{\prime}
\end{array}\right)\left[\frac{1}{\left(\epsilon \gamma_{i}\right)^{k^{\prime}+2}} \gamma\left(k^{\prime}+2, \epsilon \gamma_{i} \tau\right)+\frac{1}{\left(\epsilon \gamma_{i}\right)^{k^{\prime}+3}} \gamma\left(k^{\prime}+3, \epsilon \gamma_{i} \tau\right)\right], \\
& \left(\overline{)}=\epsilon c_{m-1} \sum_{i=1}^{m} a_{i, m}\left(\frac{\epsilon}{1+\epsilon}\right)^{l^{\prime}+1} \sum_{l^{\prime}=0}^{\gamma_{i}-1}\left(\begin{array}{c}
\gamma_{i}-1 \\
l^{\prime}
\end{array}\right)\left[p \frac{1}{\left(\epsilon \gamma_{i}\right)^{l^{\prime}+1}}\left(\gamma\left(l^{\prime}+1, a^{\prime \prime}\right)-\gamma\left(l^{\prime}+1, b^{\prime \prime}\right)\right)\right.\right. \\
& \left.+\left(\zeta^{-1}+p\right) \frac{1}{\left(\epsilon \gamma_{i}\right)^{l^{\prime}+2}}\left(\gamma\left(l^{\prime}+2, a^{\prime \prime}\right)-\gamma\left(l^{\prime}+2, b^{\prime \prime}\right)\right)+\zeta^{-1} \frac{1}{\left(\epsilon \gamma_{i}\right)^{l^{\prime}+3}}\left(\gamma\left(l^{\prime}+3, a^{\prime \prime}\right)-\gamma\left(l^{\prime}+3, b^{\prime \prime}\right)\right)\right],
\end{aligned}
$$

where $p=\left(\tau-\zeta^{-1} \tau\right), a^{\prime \prime}=\epsilon \tau^{\prime} \gamma_{i}$ and $b^{\prime \prime}=\epsilon \tau \gamma_{i}$. In the similar way, using equation(17) conditional expectation of $X_{k}$ can be written as

$$
\begin{aligned}
E\left(X_{k} \mid X_{k: m: n}>T\right) & =\int_{T}^{\infty} x f_{x_{k}}\left(x \mid X_{k: m: n}>T\right) d x \\
& =\int_{T}^{\infty} x \frac{f_{X_{k}}(x) d x}{\operatorname{Pr}\left[X_{k: m: n}>T\right]}=\int_{T}^{\infty} \frac{x f_{X_{k}}^{2}(x) d x}{\operatorname{Pr}\left[X_{k: m: n}>T\right]} \\
& =\frac{(2)}{\operatorname{Pr}\left[X_{k: m: n}>T\right]} .
\end{aligned}
$$

After simplification (Z) can be written as

$$
\begin{aligned}
\text { (2) } & =\epsilon c_{k-1} \sum_{i=1}^{k} a_{i, k} \sum_{m^{\prime}=0}^{\gamma_{i}-1}\left(\begin{array}{c}
\gamma_{i}-1 \\
m^{\prime}
\end{array}\right)\left(\frac{\epsilon}{1+\epsilon}\right)^{m^{\prime}+1}\left[R^{\prime} \zeta^{-1} \frac{1}{\left(\epsilon \gamma_{i}\right)^{m^{\prime}+1}} \Gamma\left(m^{\prime}+1, \epsilon r \gamma_{i}\right)\right. \\
& \left.+\left(\zeta^{-1}+R^{\prime}\right) \frac{1}{\left(\epsilon \gamma_{i}\right)^{m^{\prime}+2}} \Gamma\left(m^{\prime}+2, \epsilon r \gamma_{i}\right)+\zeta^{-1} \frac{1}{\left(\epsilon \gamma_{i}\right)^{m^{\prime}+3}} \Gamma\left(m^{\prime}+3, \epsilon r \gamma_{i}\right)\right],
\end{aligned}
$$

where $R=\left(\tau-\zeta^{-1} \tau\right)$ and $r=\tau+\zeta(T-\tau)$. Finally, we obtain the expression for ETTT 
given below:

$$
\begin{aligned}
\text { ETTT }= & T \sum_{j=k}^{m-1}\left[c_{j-1} \sum_{i=1}^{j} a_{i, j} \frac{\epsilon^{2}}{1+\epsilon}\left(\left(1+\frac{\epsilon \tau}{1+\epsilon}\right) e^{-\epsilon \tau}\right)^{\gamma_{j}+1} \sum_{i^{\prime}=0}^{\gamma_{i}-\gamma_{j}-2}\left(\frac{\epsilon}{1+\epsilon}\right)^{i^{\prime}}\left(\begin{array}{c}
\gamma_{i}-\gamma_{j}-2 \\
i^{\prime}
\end{array}\right)\right. \\
& {\left[\left(\frac{1}{\epsilon\left(\gamma_{i}-\gamma_{j}-1\right)}\right)^{i^{\prime}+1} \gamma\left(i^{\prime}+1,\left(\epsilon \tau\left(\gamma_{i}-\gamma_{j}-1\right)\right)\right)+\left(\frac{1}{\epsilon\left(\gamma_{i}-\gamma_{j}-1\right)}\right)^{i^{\prime}+2} \gamma\left(i^{\prime}+2,\left(\epsilon \tau\left(\gamma_{i}-\gamma_{j}-1\right)\right)\right)\right] } \\
+ & c_{j-1} \sum_{i=1}^{j} a_{i, j} \frac{\epsilon^{2}}{1+\epsilon}\left(\left(1+\frac{\epsilon \tau^{\prime}}{1+\epsilon}\right) e^{-\epsilon \tau^{\prime}}\right)^{\gamma_{j}+1} \sum_{j^{\prime}=0}^{\gamma_{i}-\gamma_{j}-2}\left(\begin{array}{c}
\gamma_{i}-\gamma_{j}-2 \\
j^{\prime}
\end{array}\right)\left(\frac{\epsilon}{1+\epsilon}\right)^{j^{\prime}} \\
& {\left[\left(\frac{1}{\epsilon\left(\gamma_{i}-\gamma_{j}-1\right)}\right)^{j^{\prime}+1}\left[\gamma\left(j^{\prime}+1, b^{\prime}\right)-\gamma\left(j^{\prime}+1, a^{\prime}\right)\right]+\left(\frac{1}{\epsilon\left(\gamma_{i}-\gamma_{j}-1\right)}\right)^{j^{\prime}+2}\left[\gamma\left(j^{\prime}+2, b^{\prime}\right)-\gamma\left(j^{\prime}+2, a^{\prime}\right)\right]\right] } \\
+ & \epsilon c_{m-1} \sum_{i=1}^{m} a_{i, m} \sum_{k^{\prime}=0}^{\gamma_{i}-1}\left(\frac{\epsilon}{1+\epsilon}\right)^{k^{\prime}+1}\left(\begin{array}{c}
\gamma_{i}-1 \\
k^{\prime}
\end{array}\right)\left[\frac{1}{\left(\epsilon \gamma_{i}\right)^{k^{\prime}+2}} \gamma\left(k^{\prime}+2, \epsilon \gamma_{i} \tau\right)+\frac{1}{\left(\epsilon \gamma_{i}\right)^{k^{\prime}+3}} \gamma\left(k^{\prime}+3, \epsilon \gamma_{i} \tau\right)\right] \\
+ & +\epsilon c_{m-1} \sum_{i=1}^{m} a_{i, m}\left(\frac{\epsilon}{1+\epsilon}\right)^{l^{\prime}+1} \sum_{l^{\prime}=0}^{\gamma_{i}-1}\left(\begin{array}{c}
\gamma_{i}-1 \\
l^{\prime}
\end{array}\right)\left[p \frac{1}{\left(\epsilon \gamma_{i}\right)^{l^{\prime}+1}}\left(\gamma\left(l^{\prime}+1, a^{\prime \prime}\right)-\gamma\left(l^{\prime}+1, b^{\prime \prime}\right)\right)\right. \\
& \left.+\left(\zeta^{-1}+p\right) \frac{1}{\left(\epsilon \gamma_{i} l^{l^{\prime}+2}\right.}\left(\gamma\left(l^{\prime}+2, a^{\prime \prime}\right)-\gamma\left(l^{\prime}+2, b^{\prime \prime}\right)\right)+\zeta^{-1} \frac{1}{\left(\epsilon \gamma_{i}\right)^{l^{\prime}+3}}\left(\gamma\left(l^{\prime}+3, a^{\prime \prime}\right)-\gamma\left(l^{\prime}+3, b^{\prime \prime}\right)\right)\right] \\
+ & \epsilon c_{k-1} \sum_{i=1}^{k} a_{i, k} \sum_{m^{\prime}=0}^{\gamma_{i}-1}\left(\begin{array}{c}
\gamma_{i}-1 \\
m^{\prime}
\end{array}\right)\left(\frac{\epsilon}{1+\epsilon}\right)^{m^{\prime}+1}\left[R^{\prime} \zeta^{-1} \frac{1}{\left(\epsilon \gamma_{i}\right)^{m^{\prime}+1}} \Gamma\left(m^{\prime}+1, \epsilon r \gamma_{i}\right)\right. \\
& \left.+\left(\zeta^{-1}+R^{\prime}\right) \frac{1}{\left(\epsilon \gamma_{i}\right)^{m^{\prime}+2}} \Gamma\left(m^{\prime}+2, \epsilon r \gamma_{i}\right)+\zeta^{-1} \frac{1}{\left(\epsilon \gamma_{i}\right)^{m^{\prime}+3}} \Gamma\left(m^{\prime}+3, \epsilon r \gamma_{i}\right)\right] .
\end{aligned}
$$

\section{Data analysis and discussions}

The elementary purpose of performing the simulation study lies in the fact that it generates repeated samples. If we compare real data study with simulation study, we will see that the real data study gives result depending on single data set while in the simulation study the results are quoted based on repeated data set. It is often unrealistic to give a general conclusion based on single data set. It motivates us to perform simulation study.

A simulation study is conducted to monitor the performance of the estimators based on the MSEs under the considered censoring scheme. The exact expression for the MSEs cannot be obtained as the estimators are not found in closed form. Therefore, the MSEs of the estimators are estimated based on a simulation study of 5,000 samples in order to get the stabilized results. The results obtained from the simulation study are summarized in tables $1-3$.

Here, It may be commemorated that the MSEs of the estimators will depend on the choice of $\epsilon, \zeta, n, m, k$, and $T$ and hence different choices have been framed for studying the effect thereof. To monitor the effect of one factor on the MSEs, the other factors are kept constant.

Table 1 shows the ML estimates, the MSEs and the estimated $95 \%$ asymptotic confidence intervals of the estimators of $\epsilon$ and $\zeta$ for accelerated GPH censored data under different removal patterns considering various choices of $\mathrm{m}, \mathrm{k}$ and $\mathrm{T}$ for fixed values of $\epsilon=0.5$ and $\zeta=1$. It may be observed from table 1 that the MSEs of the parameter $(\epsilon)$ are lesser for the removal pattern $S_{m: n}{ }^{(1)}$ compared to $S_{m: n}{ }^{(2)}$ and the MSEs under $S_{m: n}{ }^{(4)}$ are observed to be less than that for $S_{m: n}{ }^{(3)}$. Furthermore, the MSEs of the acceleration factor $(\zeta)$ are lesser under the removal pattern $S_{m: n}{ }^{(2)}$ compared to $S_{m: n}{ }^{(1)}$ and the MSEs under $S_{m: n}{ }^{(4)}$ are observed to be less than that for $S_{m: n}{ }^{(3)}$. We note that as the value of k increases, the MSEs of both $\epsilon$ and $\zeta$ decrease most of the times. Furthermore, as T decreases, the MSEs of $\epsilon$ and $\zeta$ increase under the removal pattern $S_{m: n}{ }^{(1)}$. But for the rest of removal patterns no fixed pattern is observed. 
Table 1: ML estimates, MSEs and the estimated $95 \%$ asymptotic confidence intervals of the estimators of $\epsilon$ and $\zeta$ for accelerated GPH censored data under different removal patterns considering various choices of $k$ and $T$ for fixed values of $n=100, m=80, \epsilon=0.5$ and $\zeta=1$

\begin{tabular}{|c|c|c|c|c|c|c|c|c|c|c|}
\hline $\mathrm{k}$ & $\mathrm{T}$ & $\mathrm{R}$ & $\hat{\epsilon}_{M L}$ & $M S E\left(\hat{\epsilon}_{M L}\right)$ & $\hat{\epsilon}_{L}$ & $\hat{\epsilon}_{U}$ & $\hat{\zeta}_{M L}$ & $M S E\left(\hat{\zeta}_{M L}\right)$ & $\hat{\zeta}_{L}$ & $\hat{\zeta}_{U}$ \\
\hline \multirow{8}{*}{20} & \multirow{4}{*}{20} & $S_{m: n}(1)$ & 0.5003 & 0.0018 & 0.4520 & 0.6433 & 1.1494 & 0.0429 & 0.7980 & 1.7153 \\
\hline & & $S_{m: n}^{(2)}$ & 0.5017 & 0.0024 & 0.3969 & 0.6105 & 1.1354 & 0.0381 & 0.4259 & 1.2771 \\
\hline & & $S_{m: n}{ }^{(3)}$ & 0.5013 & 0.0021 & 0.4717 & 0.6733 & 1.1235 & 0.0385 & 0.9071 & 1.7709 \\
\hline & & $S_{m: n}{ }^{(4)}$ & 0.5011 & 0.0018 & 0.3697 & 0.5653 & 1.1224 & 0.0364 & 0.8026 & 1.6602 \\
\hline & & $S_{m: n^{(1)}}$ & 0.5002 & 0.0020 & 0.4919 & 0.6830 & 1.1387 & 0.0431 & 0.3575 & 1.2669 \\
\hline & & $S_{m: n}{ }^{(2)}$ & 0.5015 & 0.0027 & 0.4152 & 0.6286 & 1.1239 & 0.0373 & 0.5629 & 1.4060 \\
\hline & 16 & $S_{m: n}{ }^{(3)}$ & 0.5010 & 0.0021 & 0.3351 & 0.5365 & 1.1418 & 0.0422 & 0.8924 & 1.7702 \\
\hline & & $S_{m: n}{ }^{(4)}$ & 0.5032 & 0.0020 & 0.4506 & 0.6471 & 1.1292 & 0.0411 & 1.0432 & 1.9071 \\
\hline \multirow{8}{*}{40} & \multirow{4}{*}{20} & $S_{m: n}{ }^{(1)}$ & 0.4998 & 0.0012 & 0.3914 & 0.5826 & 1.1333 & 0.0396 & 0.9422 & 1.8470 \\
\hline & & $S_{m: n}(2)$ & 0.5006 & 0.0023 & 0.4163 & 0.6295 & 1.1320 & 0.0362 & 0.8848 & 1.7333 \\
\hline & & $S_{m: n^{(3)}}$ & 0.5010 & 0.0019 & 0.4192 & 0.6206 & 1.1280 & 0.0379 & 0.4415 & 1.3092 \\
\hline & & $S_{m: n}{ }^{(4)}$ & 0.5001 & 0.0018 & 0.3411 & 0.5366 & 1.1365 & 0.0319 & 0.6864 & 1.5537 \\
\hline & \multirow{4}{*}{16} & $S_{m: n}{ }^{(1)}$ & 0.5020 & 0.0017 & 0.4323 & 0.6239 & 1.1281 & 0.0402 & 1.0062 & 1.9086 \\
\hline & & $S_{m: n}^{(2)}$ & 0.4994 & 0.0026 & 0.3295 & 0.5424 & 1.1362 & 0.0373 & 0.8849 & 1.7374 \\
\hline & & $S_{m: n}{ }^{(3)}$ & 0.4996 & 0.0021 & 0.4281 & 0.6292 & 1.1336 & 0.0381 & 0.7556 & 1.6269 \\
\hline & & $S_{m: n}{ }^{(4)}$ & 0.5016 & 0.0020 & 0.4530 & 0.6488 & 1.1355 & 0.0308 & 0.5996 & 1.4690 \\
\hline
\end{tabular}

In table 2, the ETTT is computed for various choices of $\epsilon$ and T under unaccelerated conditions considering removal pattern as $S_{m: n}{ }^{(4)}$. It can be noted from the table that for fixed $\mathrm{n}=100$, $\mathrm{k}=20, \mathrm{~m}=80$ and $\mathrm{T}$, as we increase the value of $\epsilon$, the ETTT decreases.

Table 2: The expected total time of the test for un-accelerated data

\begin{tabular}{|c|c|c|c|c|c|}
\hline & 0.25 & 0.50 & 0.75 & 1.00 & 1.25 \\
\hline 20 & 19.8415 & 12.8564 & 8.3706 & 6.1572 & 4.8692 \\
\hline 10 & 10.0000 & 9.8885 & 8.1156 & 6.1777 & 4.8634 \\
\hline
\end{tabular}

In table 3, keeping the censoring scheme fixed $(\mathrm{n}=100, \mathrm{k}=20, \mathrm{~m}=80)$, the ETTT is computed for accelerated GPH censored data under removal pattern $S_{m: n}{ }^{(4)}$ considering various choices of $\zeta(=1,1.5,2,2.5,3), \epsilon(=0.25,0.5,0.75,1,1.25), \mathrm{T}(=10,20)$, and $\tau(=0.5,2.5)$. It can be noted from the Table 3 that for fixed $\epsilon, \tau$, and T as $\zeta$ increases, the ETTT decreases and for fixed $\zeta$, $\tau$ and $\mathrm{T}$ as $\epsilon$ increases, the ETTT decreases. Furthermore, for fixed $\epsilon, \zeta$, and $\mathrm{T}$ as $\tau$ decreases, the ETTT also decreases. Unfortunately, no fixed pattern has been observed for studying the effect of T on ETTT under both accelerated as well as unaccelerated conditions.

To compare ETTT under accelerated and unaccelerated conditions, from the table 2 and 3, it can easily be figured out that the ETTT under unaccelerated conditions is slightly higher as compared to the ETTT under accelerated conditions for fixed T and $\epsilon$ for all choices of $\zeta$ and $\tau$.

The diagrammatic representation for the ETTT under accelerated conditions is given in Figure 2. From Figure 2, the effect of $\epsilon$ and $\zeta$ on the ETTT can be seen and it may be noted that as $\epsilon$ increases, the ETTT decreases but the rate of decrease decreases for large values of T. Similarly, as $\zeta$ increases, the ETTT decreases but the rate of decrease decreases for small values of $\tau$.

Furthermore, we considered one real-life data. The data set consists of remission times (in months) of a random sample of 128 bladder cancer patients from Lee and Wang (2003). It 
Table 3: The expected total time of the test for accelerated data

\begin{tabular}{|c|c|c|c|c|c|}
\hline & 0.25 & 0.50 & 0.75 & 1.00 & 1.25 \\
\hline & \multicolumn{5}{|c|}{$\mathrm{T}=20, \tau=2.5$} \\
\hline 1.0 & 19.8373 & 12.7963 & 8.3082 & 6.1467 & 4.9202 \\
\hline 1.5 & 18.6348 & 11.5810 & 7.9572 & 5.9823 & 4.8263 \\
\hline 2.0 & 17.5990 & 11.2243 & 7.8838 & 5.9575 & 4.7815 \\
\hline 2.5 & 17.1787 & 11.0809 & 7.8155 & 5.9544 & 4.7235 \\
\hline \multirow[t]{2}{*}{3.0} & 16.9771 & 11.0716 & 7.8038 & 5.9287 & 4.7050 \\
\hline & \multicolumn{5}{|c|}{$\mathrm{T}=10, \tau=2.5$} \\
\hline 1.0 & 10.0000 & 9.8833 & 8.1526 & 6.1751 & 4.8592 \\
\hline 1.5 & 10.0000 & 9.6265 & 7.7598 & 5.9931 & 4.8083 \\
\hline 2.0 & 9.9994 & 9.4797 & 7.6775 & 5.9599 & 4.7745 \\
\hline 2.5 & 9.9961 & 9.4459 & 7.6166 & 5.9385 & 4.7117 \\
\hline \multirow[t]{2}{*}{3.0} & 9.9887 & 9.4310 & 7.6065 & 5.9246 & 4.7092 \\
\hline & \multicolumn{5}{|c|}{$\mathrm{T}=20, \tau=0.5$} \\
\hline 1.0 & 19.8178 & 12.7749 & 8.2186 & 6.1298 & 4.8035 \\
\hline 1.5 & 17.3299 & 9.3833 & 6.4501 & 4.9952 & 4.0784 \\
\hline 2.0 & 14.3887 & 8.1594 & 5.9309 & 4.6725 & 3.8631 \\
\hline 2.5 & 12.5863 & 7.6966 & 5.7103 & 4.5641 & 3.8244 \\
\hline \multirow[t]{2}{*}{3.0} & 11.3158 & 7.3998 & 5.6159 & 4.5170 & 3.8016 \\
\hline & \multicolumn{5}{|c|}{$\mathrm{T}=10, \tau=0.5$} \\
\hline 1.0 & 9.9980 & 9.8770 & 8.1436 & 6.1658 & 4.8319 \\
\hline 1.5 & 9.9969 & 8.7770 & 6.4337 & 4.9835 & 4.0450 \\
\hline 2.0 & 9.9652 & 7.7962 & 5.8787 & 4.7002 & 3.8735 \\
\hline 2.5 & 9.7350 & 7.3056 & 5.6592 & 4.5425 & 3.8208 \\
\hline 3.0 & 9.2531 & 6.9746 & 5.5912 & 4.5337 & 3.7986 \\
\hline
\end{tabular}

has been observed that Lindley distribution can be used to analyze this data set. The fitting summary along with the ML estimate of the model parameter $\epsilon$ and its standard error denoted as $\hat{\epsilon}_{M L}$ and $\mathrm{SE}\left(\hat{\epsilon}_{M L}\right)$, is given below :

\begin{tabular}{ccccc}
\hline p-value & K-S distance & loglikelihood & AIC & BIC \\
0.0 .0832 & 0.1114 & -416.8962 & 835.7925 & 838.6445 \\
& & & & \\
$\hat{\epsilon}_{M L}$ & $S E\left(\hat{\epsilon}_{M L}\right)$ & & & \\
0.1992 & 0.0125 & & & \\
\hline
\end{tabular}

The above chart indicates that Lindley distribution fits to the above data set. For the illustration of our methodology, we have created four artificially accelerated censored data sets from the above uncensored real data set by fixing values of $k, T, m, \tau$, and four different removal patterns. Hence, we set the values of $k=40, T=12.63, m=78, \zeta=1.1$, and $\tau=2.69$ to get the accelerated censored data. The accelerated censored data along with respective censored data are given in Table 4. In Table 5, we have computed the ML estimates, the estimated $95 \%$ asymptotic variances and asymptotic confidence limits for the GPH censored data under SSPALT by considering different removal patterns as mentioned in section 5 for fixed values of $k, T, m$, and $\tau$. It can easily be seen from Table 5 that the estimates of $\epsilon$ for accelerated censored data are close to that for complete data when censoring proportion is small but they can be quite different if the censoring proportion is large. 
Table 4: Set of four artificially created censored and accelerated censored data from real data set for fixed values of $k=40, T=12.63, m=78, \zeta=1.1, \tau=2.69$ and four different removal patterns

\begin{tabular}{|c|c|c|}
\hline $\mathrm{R}$ & Censored data & Accelerated censored data \\
\hline$S_{m: n}^{(2)}$ & $\begin{array}{l}0.08,0.4,0.5,0.9,1.4,1.46,1.76,2.02,2.02,2.23, \\
2.26,2.46,2.62,2.69,2.69,2.75,3.02,3.25,3.31,3.48, \\
3.52,3.64,3.82,3.88,4.23,4.34,4.4,4.51,4.87,5.06, \\
5.17,5.32,5.34,5.41,5.41,5.62,5.85,6.25,6.54,6.76, \\
6.93,6.94,7.26,7.32,7.39,7.59,7.63,7.66,7.87,7.93, \\
8.26,8.37,8.53,8.66,9.74,10.06,10.34,10.66,10.75, \\
11.64,11.79,12.07\end{array}$ & $\begin{array}{l}0.08,0.4,0.5,0.9,1.4,1.46,1.76,2.02,2.02,2.23,2.26,2.46,2.62, \\
2.69,2.69,2.74,2.99,3.2,3.25,3.41,3.44,3.55,3.72,3.77,4.09, \\
4.19,4.24,4.34,4.67,4.84,4.94,5.08,5.1,5.16,5.16,5.35,5.56, \\
5.93,6.19,6.39,6.54,6.55,6.84,6.9,6.96,7.14,7.18,7.21,7.4, \\
7.45,7.75,7.85,8,8.12,9.1,9.39,9.64,9.94,10.02,10.83,10.96,11.22\end{array}$ \\
\hline$S_{m: n}{ }^{(4)}$ & $\begin{array}{l}0.08,0.2,0.4,0.5,0.51,0.81,0.9,1.05,1.19,1.26, \\
1.35,1.4,1.46,1.76,2.02,2.02,2.07,2.09,2.23,2.26, \\
2.46,2.54,2.62,2.64,2.69,2.69,2.75,2.83,2.87,3.02, \\
3.25,3.31,3.36,3.36,3.48,3.52,3.57,3.64,3.7,3.82, \\
4.18,4.4,4.87,5.06,5.32,5.34,5.62,5.71,5.85,6.76, \\
6.97,7.09,7.26,7.32,7.39,7.59,7.63,8.53,8.66,9.47, \\
9.74,10.06,10.75,11.25,11.64,11.79,12.02,12.03, \\
12.07\end{array}$ & $\begin{array}{l}0.08,0.2,0.4,0.5,0.51,0.81,0.9,1.05,1.19,1.26,1.35,1.4,1.46, \\
1.76,2.02,2.02,2.07,2.09,2.23,2.26,2.46,2.54,2.62,2.64,2.69, \\
2.69,2.74,2.82,2.85,2.99,3.2,3.25,3.3,3.3,3.41,3.44,3.49,3.55, \\
3.61,3.72,4.04,4.24,4.67,4.84,5.08,5.1,5.35,5.44,5.56,6.39, \\
6.58,6.69,6.84,6.9,6.96,7.14,7.18,8,8.12,8.85,9.1,9.39,10.02, \\
10.47,10.83,10.96,11.17,11.18,11.22\end{array}$ \\
\hline
\end{tabular}

Table 5: ML estimates, Asymptotic variances and the estimated $95 \%$ asymptotic confidence limits for parameters $\epsilon$ and $\zeta$ for the GPH censored data under SSPALT by considering different removal patterns for fixed values of $k=40, T=12.63, m=78, \zeta=1.1$ and $\tau=2.69$

\begin{tabular}{ccccccccc}
\hline $\mathrm{R}$ & $\hat{\epsilon}_{M L}$ & $\operatorname{var}\left(\epsilon_{M L}\right)$ & $\hat{\epsilon}_{L}$ & $\hat{\epsilon}_{U}$ & $\hat{\zeta}_{M L}$ & $\operatorname{var}\left(\hat{\zeta}_{M L}\right)$ & $\hat{\zeta}_{L}$ & $\hat{\zeta}_{U}$ \\
\hline$S_{m: n}{ }^{(1)}$ & 0.2365 & 0.0008 & 0.1810 & 0.2921 & 1.1284 & 0.0468 & 0.7042 & 1.5526 \\
$S_{m: n}{ }^{(2)}$ & 0.2286 & 0.0012 & 0.1597 & 0.2976 & 1.0258 & 0.0519 & 0.5794 & 1.4722 \\
$S_{m: n}{ }^{(3)}$ & 0.2406 & 0.0010 & 0.1791 & 0.3021 & 1.0085 & 0.0383 & 0.6249 & 1.3921 \\
$S_{m: n}{ }^{(4)}$ & 0.2390 & 0.0008 & 0.1835 & 0.2944 & 1.0408 & 0.0415 & 0.6418 & 1.4399 \\
\hline
\end{tabular}



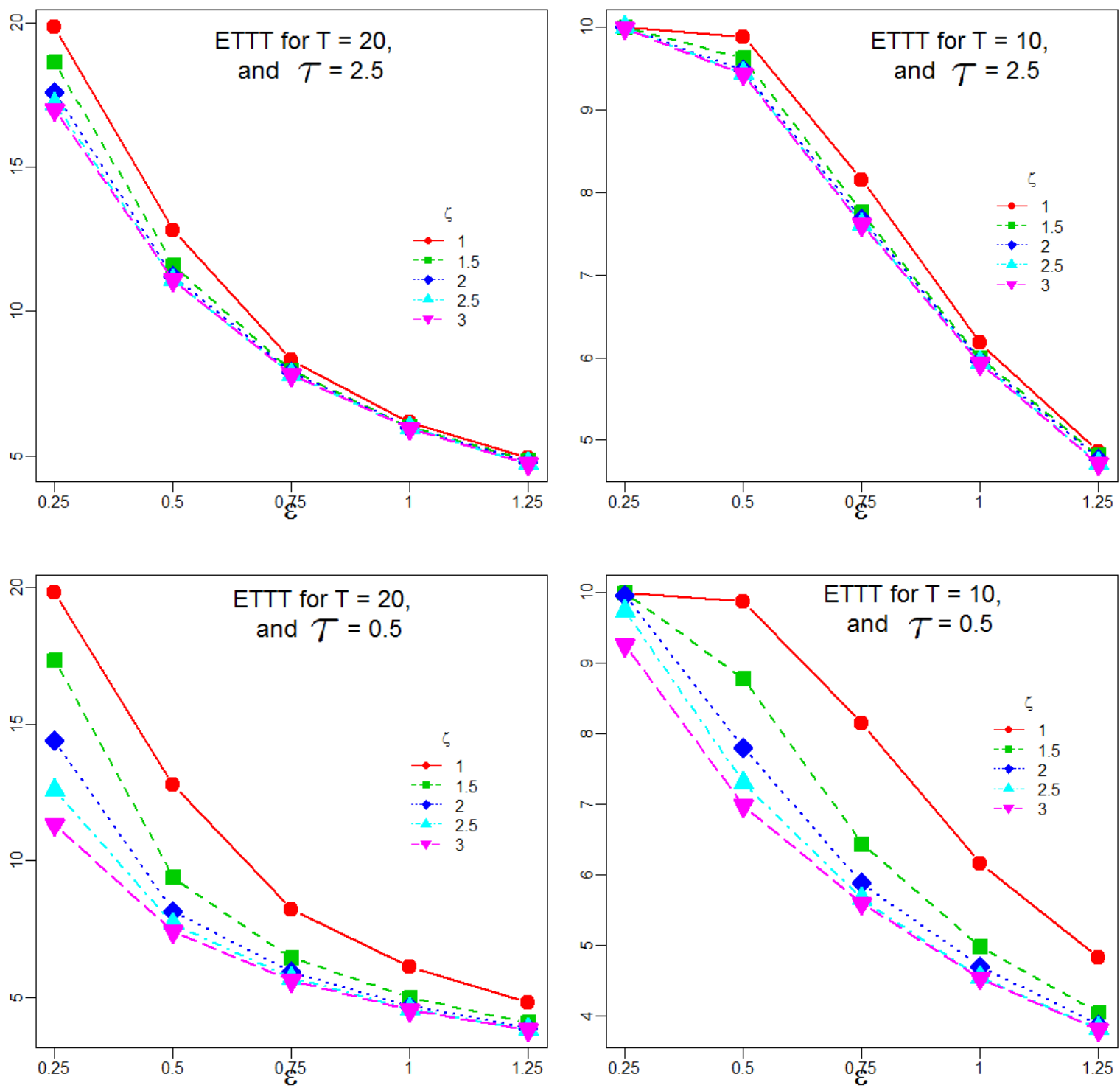

Figure 2: The ETTT for various choices of $\epsilon, \zeta, \mathrm{T}$ and $\tau$

\section{Conclusion}

The article highlights a sampling procedure for a life testing experiment called step stress partially accelerated life test with the GPH censoring scheme. A few prongs of the results have been presented as:

1. The ETTT under this sampling procedure has been investigated. It has been observed that $\zeta$ and $\tau$ are scandalously controlling the duration of the test. For large values of $\zeta$, the ETTT is noted to be smaller however, as $\tau$ increases the ETTT increases, keeping other factors fixed. Thus, a reduction in the ETTT is expected for large values of $\zeta$ and small values of $\tau$.

2. It has also been observed that the removal patterns have different effect on the estimate of the parameter and the acceleration factor as illustrated above. For the estimation of parameter of the distribution, one can use removal pattern $S_{m: n}{ }^{(1)}$ in place of $S_{m: n}{ }^{(2)}$ and $S_{m: n}{ }^{(4)}$ in place of $S_{m: n}{ }^{(3)}$. On the other hand, if one is interested in estimation of the acceleration factor, one can prefer removal pattern $S_{m: n}{ }^{2)}$ to $S_{m: n}{ }^{(1)}$ and $S_{m: n}{ }^{(4)}$ to $S_{m: n}{ }^{(3)}$. Since, for the large values of $k$, the MSEs of estimators of $\epsilon$ and $\zeta$ decrease. Therefore, it is suggested to keep the value of $k$ high. Similarly, we suggest to keep the 
value of $T$ small.

3. It can also be seen that the ETTT under un-accelerated conditions is slightly higher as comparison to the ETTT under accelerated conditions for fixed $T$ and $\epsilon$ for all choices of $\zeta$ and $\tau$. Therefore, we may conclude that acceleration is beneficial for the test.

The accelerated life test is often a default choice in a situation where the reliability of the products is relatively high. Therefore, such problems can be speedily settled with the help of an accelerated life test model. The present work is done where the acceleration of the lifetime data is done at a single step. But the work can be extended by accelerating the lifetime at multiple steps. It would be of a great interest to find the optimum inspection times of SSPALT with the GPH censored data. Moreover, the Bayesian counterpart of the current work can also be attempted and the results can be compared with the classical one. The paper can also be extended by applying SSPALTs to other censoring schemes also, such as interval censoring scheme.

\section{Acknowledgment}

We gratefully thank the editor, Dr. Matthias Templ and anonymous referees for their valuable comments on an earlier version that lead to the improved final version.

\section{References}

Abd-Elfattah AM, Hassan AS, Nassr SG (2008). "Estimation in Step-Stress Partially Accelerated Life Tests for the Burr Type-XII Distribution Using Type-I Censoring." Statistical Methodology, 5(6), 502-514.

Bai DS, Chung SW (1992). "Optimal Design of Partially Accelerated Life Tests for the Exponential Distribution under Type-I Censoring." IEEE Transactions on Reliability, 41(3), 400-406.

Balakrishnan K (1996). Exponential Distribution: Theory, Methods and Applications. CRC press.

Balakrishnan N (2007). "Progressive Censoring Methodology: An Appraisal." Test, 16(2), 211.

Balakrishnan N, Aggarwala R (2000). Progressive Censoring: Theory, Methods, and Applications. Springer Science \& Business Media.

Cakmakyapan S, Ozel G (2016). "The Lindley Family of Distributions: Properties and Applications." Hacettepe Journal of Mathematics and Statistics, 46, 1-27.

Childs A, Chandrasekar B, Balakrishnan N (2008). "Exact Likelihood Inference for an Exponential Parameter under Progressive Hybrid Censoring Schemes." In Vonta F., Nikulin M., Limnios N., Huber-Carol C. (eds) Statistical Models and Methods for Biomedical and Technical Systems. Statistics for Industry and Technology, pp. 319-330. Springer Birkhäuser Boston.

Childs A, Chandrasekar B, Balakrishnan N, Kundu D (2003). "Exact Likelihood Inference Based on Type-I and Type-II Hybrid Censored Samples from the Exponential Distribution." Annals of the Institute of Statistical Mathematics, 55(2), 319-330.

Cho Y, Sun H, Lee K (2015). "Exact Likelihood Inference for an Exponential Parameter under Generalized Progressive Hybrid Censoring Scheme." Statistical Methodology, 23, 18-34. 
DeGroot MH, Goel PK (1979). "Bayesian Estimation and Optimal Designs in Partially Accelerated Life Testing." Naval Research Logistics Quarterly, 26(2), 223-235.

Eissa FH, Ahmed HH, Wu SJ (2014). "Statistical Inferences of the Exponentiated Weibull Model under Type II Progressively Censored Data with Random Removals." Wulfenia, 21(3), 335-348.

Epstein B (1954). "Truncated Life Tests in the Exponential Case." The Annals of Mathematical Statistics, 25(3), 555-564.

Ismail AA (2012). "Estimating the Parameters of Weibull Distribution and the Acceleration Factor from Hybrid Partially Accelerated Life Test." Applied Mathematical Modelling, 36(7), 2920-2925.

Kundu D, Joarder A (2006). "Analysis of Type-II Progressively Hybrid Censored Data." Computational Statistics \& Data Analysis, 50(10), 2509-2528.

Lee ET, Wang J (2003). Statistical Methods for Survival Data Analysis, volume 476. John Wiley \& Sons.

Lindley DV (1958). "Fiducial Distributions and Bayes' Theorem." Journal of the Royal Statistical Society. Series B (Methodological), pp. 102-107.

Nelson WB (1990). Accelerated Life Testing: Statistical Models, Data Analysis and Test Plans. Wiley Series in Probability and Statistics.

\section{Affiliation:}

Aakriti Pandey

Department of Statistics

Institute of Science

Banaras Hindu University

Varanasi, India-221005

E-mail: akritibhu@gmail.com

Arun Kaushik

Department of Statistics, Institute of Science

Banaras Hindu University

Varanasi, India-221005

E-mail: arundevkauhsik@gmail.com

URL: http://arun-kaushik.github.io/

\section{Austrian Journal of Statistics}

published by the Austrian Society of Statistics

Volume 50

January 2021 http://www.ajs.or.at/

http://www.osg.or.at/

Submitted: 2019-08-13

Accepted: 2020-01-21 